\title{
Ueher die Durchtrittsweise des vorausgehenden Schädels durch den Eingang eines einfach platten Beckens.
}

Von

\author{
Dohrn.
}

(Mit 1. Molzschnitt.)

In einem kürzlich erschienenen Aufsatze ,zum Geburtsmechanismus beim engen Becken" "l) behauptet Haselberg, dass das enge Becken an sich den nachfolgenden Kopf leichter passiren lasse, als den vorausgehenden. Bei dem Versuche, diesen Satz zu. demonstriren, geht er von der Annahme aus, dass der vorausgehende Kopf mit seinem ,grossen schiefen Durchmesser“" 2) die Conjugata zu passiren habe, während dagegen der nachfolgende Kopf gerade, d. i. mit beiden Seitenhälften gleichmässig, auf die Conjugata hinabrücke und diese dann mit seinem queren Durchmesser, welcher kleiner ist als jener grosse schiefe, passire.

Dieser Darstellung zufolge würde bei den Schädellagen das Promontorium das hinterwärts belegene Scheitelbein in einer Linie

1) Berliner Beiträge f. Geburtsh. II. Bd. 3. Heft.

2) H. nennt so einen Durchmesser, welcher von der höchsten Stelle der Sutura squamosa einer Seite zu der vom anderen Tuber parietale nach vorn gehenden Leiste in der Ebene des vorderen Querdurchmessers gezogen wird. 
'streifen, welche von der' Gegend der grossen Fontanelle aus parallel der Kranznaht über den Knochen hinwegzieht.

Der beschriebene Mechanismus kommt vor; wenn man denselben aber für den gewöhnlichen oder gar nothwendigen halten wollte, so wäre das durchaus irrthümlich. Nur bei besonders günstigen Umständen wixd der Kopf in dieser Weise hindurchtreten, für gewöhnlich schlägt die Natur einen anderen Weg ein, welcher rascher und besser zum Ziele führt, und jedenfalls hat man keinen Grund, jenen seltenen und ungünstigen Mechanismus zur Voraussetzung seiner. therapeutischen Folgerungen zu machen.

Michaelis hat, wie allgemein bekannt, gelehrt und erklärt, dass bei dem einfach platten Becken der Kopf sich quer in den Beckeneingang stellt, die Pfeilnaht näher dem Promontorium als der Schossfuge, die grosse Fontanelle näher dem Vorberg als die kleine. Diese Stellung ist diejenige, in welcher der Kopf sich im Beckeneingange fixirt, in welcher das grösste Segment des Schädels in den Beckeneingang hinabzureichen vermag und in welcher seine Configuration, wie sie das Becken fordert, am besten erfolgt.

Hierüber sind Alle einig und in der That bieten langdauernde Geburten bei platten Becken hinreichende Gelegenheit, diese Einstellung zu beobachten.

Eine andere Sache ist es aber mit dem Mechanismus, vermittels dessen der Kopf den Beckeneingang passirt. Bei der vorhin angegebenen Stellung ruht der Kopf vorne mit der Gegend der Schuppennaht auf der Schossfuge, hinten mit einer der grossen Fontanelle benachbarten Stelle aúf dem Promontorium. Während rechts und links Stirn und Hinterhaupt völlig oder mindestens doch vergleichsweise frei sind, werden nun die in der Conjugata befindlichen Schädelstellen unter eintretender Configuration des Kopíes, stärkerer Vorwölbung des vorne belegenen, Abflachung des hinteren Scheitelbeines, fester in die Conjugata eingezwängt.

Aus dieser Stellung könnte der Kopf in einer doppelten Weise in die Beckenhöhle hinabrücken, entweder so, dass das. Promontorium in der Richtung gegen das Ohr hin über das Scheitelbein weg geht oder so, dass die Spur desselben die Kranznaht von oben hinten nach unten vorne kreuzt. Michaelis glaubte, dass der erstere Mechanismus der gewöhnliche sei, und ihm ist das von späteren Autoren häufig nachgeschrieben worden; sieht man 
aber seine eigenen Fälle durch, so gelangt man, gleichwie bei theoretischer Untersuchung der hier obwaltenden mechanischen Verhältnisse, zu der Einsicht, dass dieser Durchtrittsmechanismus ein ausnahmsweiser, nicht der gewöhnliche ist.

Schon bevor diese Zeilen geschrieben, machte Spiegelberg auf der Naturforscherversammlung in Wiesbaden darauf aufmerksam, dass bei den in Rede stehenden Fällen eine eintretende Senkung des Hinterhauptes den Durchtritt des Kopfes vorbereite und ermögliche. ') Diese Auffassung entspricht meiner Erfahrung durchaus. Zur Erläuterung des Vorganges möge aber folgendes dienen.

Wenn der Kopf sich mit seinem, ,kleinen schiefen Durchmesser" 2), wie Haselberg, Michaelis folgend, angegeben, in die Conjugata hineingestellt hat, so wird er sich um diesen nunmehr fixirten Durchmesser leichter rotiren, als dass die Drehung um seinen geraden Durchmesser, dessen Endpunkte gar nicht fixirt sind, erfolgen sollte. Der ersteren Bewegung, welche mit Senkung der Stirn oder des Hinterhauptes eñden muss, steht, wenn es sich um einfach platte oder um platte Becken mit nur geringer allgemeiner Verengerung handelt, seitens des Beckens Nichts entgegen, der letzteren Bewegung dagegen ist die Prominenz des hinteren Scheitelbeines erschwerend oder absolut hinderlich. Trifft im letzteren Falle das Promontorium auf einen stärkeren Widerstand des Scheitelbeines, welcher sich nicht durch Abflachung oder Impression ausgleicht, so wird entweder spontan oder durch einfache Lagerung der Gebärenden auf die entsprechende Seite der Art Abhülfe erreicht, dass die kleine Fontanelle sich senkt und nun das Promontorium die Kranznaht nach vorn schneidend an das schmälerere Stirnbein gelangt. Hierfür ein Beispiel.

1) Ein Resumé meiner hier vom Verf. angezogenen Beobachtungen findet sich schon in einem vor der Brit. Med. Assoc. im August d. J. gehaltenen Vortrage (s. Brit. Med. Joumal, 18. October 1873). Die Wiesbadener Bemerkungen werden mit den übrigen in der dortigen gynäkologischen Section gehaltenen Vorträgen und Discussionen im Archiv baldigst mitgetheilt werden.

Spiegelberg.

2) Als kleinen schiefen Durchmesser bezeichnet $H$. eine Linie, welche die höchste Stelle der Schuppennaht mit einem Punkte des vorderen oberen Winkels des anderen Scheitelbeines, welcher circa $14 \mathrm{Mm}$. von beiden Nähten entfernt ist, verbindet. 
A. H., Erstgebärende, mit plattem, gering allgemein rerengtem Becken. Conjug. vera 7,8 Cm. Künstliche Frühgeburt in der 35 . Woche. Nach 24stündiger Anwendung eines intrauterinen Bougies Wasserabfluss bei $3 \mathrm{Cm}$. weitem Muttermunde. Der in 1. Schädellage befindliche Kopf stand beim Wasserabfluss quer im Beckeneingange, die Pfeilnaht circa $2 \mathrm{Cm}$. vom Promontorium entfernt, die grosse Fontanelle nahe dem letzteren, das hintere Scheitelbein untergeschoben, die kleine Fontanelle hoch links zur Seite. Unter sehr kräftigen Wehen zeigte sich nach $1^{1 / 2}$ Stunden das hintere Scheitelbein etwas weiter am Promontorium herabgepresst und die kleine Fontanelle etwas mehr gesenkt. Der Kreissenden wird nun die linke Seitenlage gegeben. Nach 15 Minuten kommt der Kopf zum Einschneiden. Lebendes Kind von $2645 \mathrm{Gm}$. Gewicht. Querer Kopfdurchmesser 8,2, gerader 11,4, diagonaler $13 \mathrm{Cm}$. Diffuse Kopfgeschwulst über dem rechten Scheitelbeine. Die ganze linke Schädelfï̈che ist abgeflacht und nach vorn geschoben, das Scheitelbein unter-, das Stirnbein übergeschoben. Die linke Schuppennaht klafft stärker als die rechte. Auf dem linken Scheitelbeine ein $0,5-1,0 \mathrm{Cm}$. breiter rother Druckstreif, der in der Nähe der grossen Fontanelle und Pfeilnaht beginnt, von da nach dem Tuber parietale hinzieht, sich hier unter einem fast rechten Winkel nach vorn wendet und in einer tiefen Furche zwischen Schuppen- und Kranznaht endigt (s. Fig.).

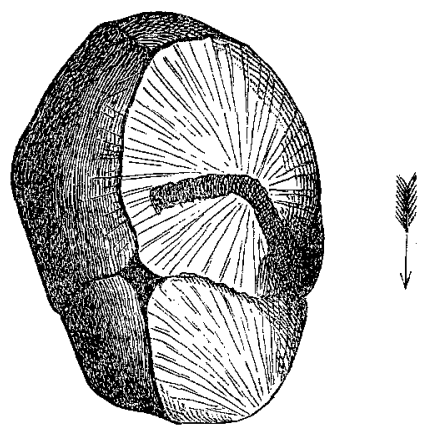

Hier war demnach der Vorgang so gewesen, dass das linke Scheitelbein bei seinem Herabrücken am Promontorium Widerstand gefunden hatte, und dieser Widerstand nun nicht etwa durch rinnenförmige Einbiegung der prominentesten Partie des Scheitelbeines ausgeglichen wurde, sondern vielmehr eine Senkung des Hinterhauptes eintrat, welche, durch entsprechende Seitenlagerung der Kreissenden unterstützt, rasch das Hinabgleiten des Kopfes am Promontorium zur Folge hatte. Die hervorragendste Stelle des Scheitelbeines ging somit gar nicht durch die Conjugata hindurch, das Tuber parietale glitt zwar in der Nähe, aber doch 
oberhalb des Promontoriums nach links in den Beckeneingang hinab.

Den so beschriebenen Mechanismus halte ich für den gewöhnlichen, und wenn das Missverhältniss hochgradig ist und die Schädelknochen derb, sogar für den allein möglichen. Dagegen wird ein Fortrücken des Promontoriums in der anfänglichen, senkrecht auf der Pfeilnaht stehenden Richtung nur dann stattfinden, wenn die Wehen ungewöhnlich stark, das Scheitelbein gut compressibel, das räumliche Missverhältniss nicht bedeutend ist. Ich könnte manche Fälle anführen, welche das beweisen, in denen gleich von Anfang an in gerader Linie oder in leicht gekrümmten Bogen die Druckspur des Promontorium nach vorn und unten über das Scheitelbein weg ging, ich beschränke mich aber auf die Eine vorstehende Mittheilung, um den Mechanismus auch dieser Geburtsfälle damit zu kennzeichnen.

Wenn man bisher oft von der rinnenförmigen Einbuchtung des Margo coronalis des hinterwärts belegenen Scheitelbeins, kaum jemals dagegen von der Senkung des Hinterhauptes und der diesem Vorgange entsprechenden Druckspur des Promontoriums gesprochen hat, so liegt der Grund wohl vorzugsweise darin, dass die Senkung des Hinterhauptes ein rasch vorïbergehender Vorgang ist, welcher sich auch durch die Druckspuren am Kopfe wenig markirt, während dagegen die Anstemmung des hinteren Scheitelbeines an das Promontorium durch den touchirenden Finger und später an der Druckspur hinreichend beobachtet werden kann. Kaum ist es auch wohl anders zu erklären, dass selbst ein Mann, wie Michaelis, über die in Rede stehenden Fälle angiebt, die meisten Druckstellen hätten sowohl auf dem Scheitelbeine als auf dem Stirnbeine gelegen ${ }^{1}$ ), und dennoch andererseits (1. c. §. 218) behauptet, das Promontorium rücke gewöhnlich gerade gegen das $\mathrm{Ohr}$ fort.

Wäre Letzteres der Fall, so müsste auch die rinnenförmige Finbiegung des Margo coronalis viel häufiger vorkommen, als das die Beobachtung ergiebt. Litzmann berechnet für diese'Gestaltveränderung des Knochens nur eine Häufigkeit von 7,3\% und begreift unter diese Zahl noch die rinnenförmigen Einbiegungen des Margo sagittalis und temporalis mit. ${ }^{2}$ ) In der Schä-

1) s. das enge Becken §. 270.

2) s. Volkmann's klinische Vorträge Nr. 23. 
delsammlung der Marburger Entbindungsanstalt, welche eine reiche Auswahl von Schädeln bietet, die platte Becken passirt haben, ist die rinnenförmige Einbiegung des Margo coronalis durch kein einziges Exemplar vertreten.

Ich mache hier noch auf eine andere Wahrnehmung aufmerksam, welche zur Controle der Richtung dienen kann, in welcher der Kopf das Promontorium passirte. Bekanntlich findet man oft bei Köpfen. welche ein plattes Becken passirt haben, die Scheitelbeine in sagittaler Richtung gegen einander verschoben. Ich habe das Gesetz, nach welchem sich die Richtung dieser Verschiebung bestimmt, so gefasst, dass das hintere Scheitelbein bei vorausgehender grosser Fontanelle vom Promontorium zurück, bei vorausgehender kleiner Fontanelle dagegen nach vorn geschoben werde. ${ }^{1}$ ) Litzmann bemerkt (1. c.), er habe das der Mehrzahl nach bestärigt gefunden, doch fehle es nicht an Ausnahmen. Es ist nach obigem klar, wie diese Ausnahmen zu deuten sind. Fand der Kopf einen stärkeren Widerstand am Promontorium nur vor der Senkung des Hinterhauptes, so weicht das betreffende Scheitelbein zurück, fand er einigen Widerstand auch noch nach Eintritt jener Senkung, so wird das Scheitelbein nach vorn geschoben. Das von mir aufgestellte Gesetz bleibt daher gültig, die Verschiedenheit der Ergebnisse beruht nur eben in der Verschiedenheit des Mechanismus, mittels dessen der Kopf den Beckeneingang passirt. So hat auch in dem von mir oben mitgetheilten Geburtsfalle die nach vorn verlaufende Druckspur des Promontoriums den Ausschlag gegeben für die Verschiebung des linken Scheitelbeines nach vorn; wäre das Promontorium dagegen in der anfänglichen Richtung weiter über das Scheitelbein hinweggegangen, so wäre dieser Knochen auch gewiss nach hinten geschoben worden.

Nimmt man einen skelettirten Schädel von geeigneter Grösse und versucht, denselben durch den Eingang eines einfach platten Beckens hindurchzuschieben, so wird man bald finden, dass der vorausgehende Schädel nur dann den Beckeneingang passirt, wenn man ihn zunächst mit seinem ,kleinen schiefen Durchmesser“ in die Conjugata stellt und dann, um diese Drehaxe rotirend, das Hinterhaupt durch den Beckeneingang hinabsenkt. Für die Verhältnisse an der Lebenden giebt ein solcher Versuch allerdings

1) s. Bericht über die Naturforscherversammlung in Giessen 1864. 
88 Dohrz, Ueber die Durchtrittsweise des vorausgehenden Schädels u. s. w.

insofern keinen ganz richtigen Ausdruck, als bei solchen präparirten Schädeln die Compressibilität des Knochens wegfällt, żur Beurtheilung der Einstellung dagegen, insoweit dieselbe lediglich von der Form und Grösse der betreffenden Theile abhängt, ist derselbe lehrreich. ${ }^{1}$ )

Das Resultat meiner Erörterungen ist also das, dass bei dem einfach platten Becken keineswegs die grösste Wölbung des hinteren Scheitelbeines, des Tuber parietale und dessen nächste Nachbarschaft am Promontorium hinabzurücken braucht, sondern vielmehr das Promontorium die Kranznaht nach vorn und unten zu schneiden pflegt, dass es daher falsch ist, durch die Annahme jenes ersteren selteneren und ungünstigen Mechanismus einen Vortheil der Wendung bei engem Becken begründen zu wollen. Liegt der Schädel vor, so haben wir vielmehr Grund, dass wir, sobald derselbe fest auf die Conjugata herabgepresst ist, die Senkung des Hinterhauptes durch geeignete Anordnungen befördern, und sobald dieselbe erreicht ist, werden wir bei engen Becken mittlern Grades den Kopf in einer mechanisch sehr günstigen Weise den Beckeneingang passiren sehen.

1) In meinen Vorlesungen wende ich seit Jahren zur Demonstration dieser Einstellungen Tafeln von starker Pappe an, in welchen ein Beckeneingang von bestimmter Weite und Form ausgeschnitten ist. Sucht man durch die herausgeschnittene Oefinung einen Schädel hindurchzuschieben, so gelangt man zu derselben Einstellung, wie wir sie an der Lebenden beobachten. Zum Belege folgende Maassangaben. Man schneide in einer Papptafel einen Beckeneingang ron folgender Form aus:

$\begin{array}{lr}\text { Conjugata } & 7,7 \mathrm{Cm} . \\ \text { Querer Durchmesser } & 13,6 \quad " \\ \text { Schräger ", } & 10,5 \quad,\end{array}$

und suche nun einen Kopf in Schädellage durchzuschieben, welcher folgende Maasse hat:

$\begin{array}{lr}\text { grosser Querdurchmesser } & 9,4 \mathrm{Cm} \text {. } \\ \text { kleiner } & 8,0 \quad " \\ \text { gerader Durchmesser } & \mathbf{1 2 , 0} " \\ \text { diagonaler } \quad, & 12,9 \quad "\end{array}$

so wird man finden, dass dieser Schädel in keiner anderen als der von mir angegebenen Weise durch den Beckeneingang hindurchzuschieben ist. 